\title{
Microarray profiles of ex vivo expanded hematopoietic stem cells show induction of genes involved in noncanonical Wnt signaling
}

\author{
D.L. Zanette ${ }^{1,2}$, J.C.C. Lorenzi ${ }^{1,2}$, R.A. Panepucci, ${ }^{1,2}$, A.R.D. Santos ${ }^{1,2}$, \\ G.A. Molfetta ${ }^{1,2}$, A.G. Araujo ${ }^{2,3}$, W.A. Silva Junior ${ }^{1,2}$ and M.A. Zago ${ }^{2,3}$ \\ ${ }^{1}$ Departamento de Genética, Faculdade de Medicina de Ribeirão Preto, \\ Universidade de São Paulo, Ribeirão Preto, SP, Brasil \\ ${ }^{2}$ Centro de Terapia Celular, Centro Regional de Hemoterapia, \\ Ribeirão Preto, SP, Brasil \\ ${ }^{3}$ Departamento de Clínica Médica, Faculdade de Medicina de Ribeirão Preto, \\ Universidade de São Paulo, Ribeirão Preto, SP, Brasil \\ Corresponding author: D.L. Zanette \\ E-mail: zanette@usp.br
}

Genet. Mol. Res. 12 (2): 1691-1697 (2013)

Received October 1, 2012

Accepted January 17, 2013

Published May 16, 2013

DOI http://dx.doi.org/10.4238/2013.May.15.1

\begin{abstract}
The low number of hematopoietic stem cells (HSC) in umbilical cord blood (UCB) is directly related to increased risk of transplant failure. Effective ex vivo expansion of HSC has been tried for many years, with conflicting results because of the inability to reproduce in vitro HSC proliferation in the same way it occurs in vivo. We compared freshly isolated HSC with their expanded counterparts by microarray analysis and detected activation of the noncanonical Wnt (wingless-type MMTV integration site family) pathway. Study of early alterations during ex vivo UCB-HSC expansion could contribute to improvement of ex vivo expansion systems.
\end{abstract}

Key words: Umbilical cord blood; Hematopoietic stem cell; Microarrays; Ex vivo expansion 


\section{INTRODUCTION}

Bone marrow hematopoietic stem cells (HSC) are traditionally used in hematopoietic transplantation, but HSC obtained from umbilical cord blood (UCB) (Kelly et al., 2009) have some advantages, such as availability from cryopreserved units and decreased risk of graftversus-host disease. However, the low number of HSC in UCB is directly related to increased risk of graft failure and delayed engraftment and immune reconstitution. Effective in vitro expansion of HSC has been long tried, with conflicting reports of success (Gilmore et al., 2000; McNiece et al., 2000; Pranke et al., 2005; Walenda et al., 2011; Auvray et al., 2012). The failure of these protocols may be due to the inability to reproduce in vitro the combination of factors that occurs in vivo, where simultaneous HSC proliferation and maintenance of long-term hematopoietic reconstitution is achieved (Astori et al., 2001; Blank et al., 2008).

The transplantation of two UCB units, one ex vivo expanded and one unmanipulated, has proven to be effective. A promising clinical trial using ex vivo expanded UCB-HSC in the presence of the Notch ligand Delta1 results in enhanced initial myeloid engraftment, suggesting that these cultured cells promote long-term engraftment of the unmanipulated UCB unit that is concomitantly infused (Delaney et al., 2010).

The summarized gene expression profiles of expanded HSC and their unmanipulated counterparts reported here suggest activation of the noncanonical Wnt pathway. Study of these early alterations during expansion may contribute to a better understanding of HSC biology and to improvement of ex vivo expansion systems.

\section{MATERIAL AND METHODS}

CD34 ${ }^{+}$cells were obtained from 13 UCB samples using magnetic beads (MACS, Miltenyi Biotec), after informed consent of the mother. Samples were split in 2 freshly isolated $\mathrm{CD}^{+} 4^{+}$cells (t0) and 3-day ex-vivo expanded cells ( $\mathrm{t} 3$ ), which were used for flow cytometry and RNA extraction. A FACScan (Becton Dickinson, San Diego, CA, USA) system was used to determine the starting $\mathrm{CD} 34^{+}$purity (always $>88 \%$ ), $\mathrm{CD} 34^{+} / \mathrm{CD} 90^{+}, \mathrm{CD} 34^{+} / \mathrm{CD} 38^{\text {neg }}$, and cell viability by annexin- $\mathrm{V}$ and propidium iodide. All analyses were performed at $\mathrm{t} 0$ and $\mathrm{t} 3$, counting 10,000 events, with the Cellquest software (Becton Dickinson).

Cells were cultured under serum-free conditions, in FreeStyle 293 Expression Medium (Invitrogen, Carlsbad, CA, USA), supplemented with 1\% human albumin and a cocktail of cytokines, based on previous reports (Pranke et al., 2005; Kelly et al., 2009): $50 \mathrm{ng} / \mathrm{mL}$ flt-3 ligand, $35 \mathrm{ng} / \mathrm{mL}$ thrombopoietin, $50 \mathrm{ng} / \mathrm{mL}$ stem cell factor, and $10 \mathrm{ng} / \mathrm{mL}$ interleukin-6.

Total RNA was isolated with TRIzol ${ }^{\circledR}$ LS Reagent (Invitrogen), following manufacturer recommendations. Gene expression profiles were obtained using Amersham CodeLink UniSet Human I BioArrays (GE Amersham Biosciences, Piscataway, NJ, USA) platform. We used 2 pools of unmanipulated $\mathrm{CD} 34^{+}$cells ( $\mathrm{t} 0$ ) and 2 pools of expanded cells ( $\mathrm{t} 3$ ), each pool consisting of 4 different samples. The fluorescence values were normalized (75th percentile) and the foldchanges were averaged between the 2 pools from $\mathrm{t} 3$ and $\mathrm{t} 0$.

Validation of microarray results was performed with reverse transcribed cDNAs and $\operatorname{TaqMan}^{\circledR}$ probes in an ABI Prism 7500 Detection System (Applied Biosystems). Normalization was based on the geometric mean of the Ct values for 2 endogenous genes, ACTB and GUSB. The $2^{\Delta \Delta \mathrm{Ct}}$ method (Pfaffl, 2001) was used to compare gene expression profiles of $\mathrm{t} 3$ and $\mathrm{t} 0$ cells, 
with the latter used as the calibrator. Statistical analyses were performed using the GraphPad software (nonparametric, one-tailed Mann-Whitney test, 95\% confidence interval, $\mathrm{P}<0.05$ ).

\section{RESULTS}

The expansion protocol resulted in an average 2.5 -fold increase in the absolute number of $\mathrm{CD} 34^{+}$cells $(\mathrm{P}=0.0001$; Figure $1 \mathrm{~A})$ and most cells preserved the surface $\mathrm{CD} 34^{+}$expression in $\mathrm{t} 3$ (Figure 1B). The absolute number of $\mathrm{CD} 34^{+} / \mathrm{CD} 38^{-}$cells increased 7.2 -fold $(\mathrm{P}=0.0001$; Figure $2 \mathrm{~A})$. The percentage of $\mathrm{CD} 34^{+} / \mathrm{CD} 38^{-}$cells was also increased $(\mathrm{P}=0.012$; Figure $2 \mathrm{~B})$. The absolute number of $\mathrm{CD} 34^{+} / \mathrm{CD} 90^{+}$cells showed an average 5.9 -fold increase, although not significant $(\mathrm{P}=0.15)$ and there was no induction of cell death at $\mathrm{t} 3$ (data not shown).
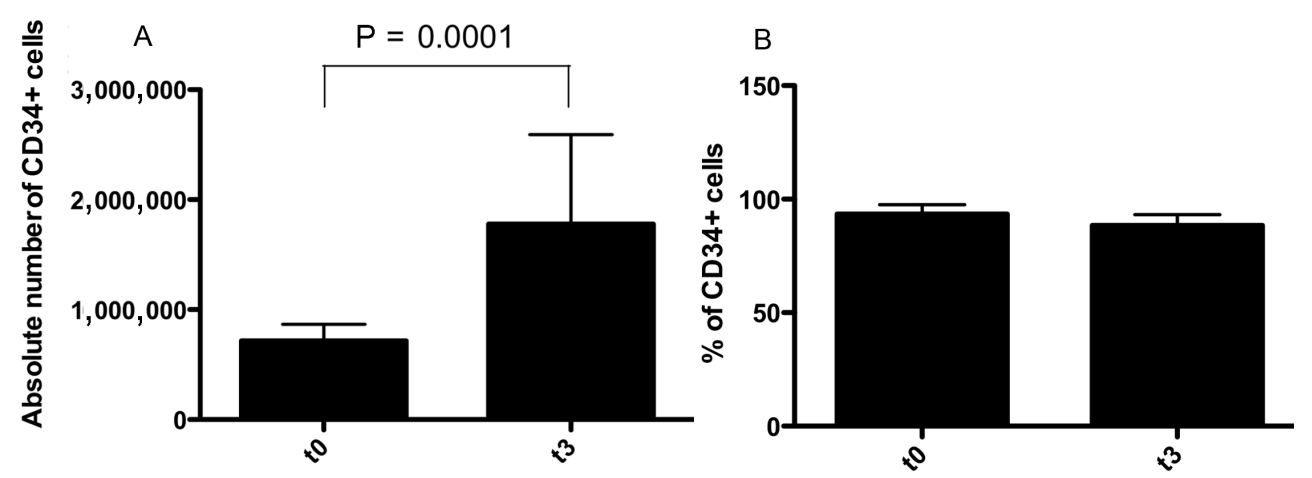

Figure 1. $\mathrm{CD}_{3} 4^{+}$contents in unmanipulated $(\mathrm{t} 0)$ and expanded $(\mathrm{t} 3)$ cells and validation of microarray expression data of t0 and $\mathrm{t} 3$ cells. A. Absolute number of $\mathrm{CD} 34^{+}$cells at t0 and $\mathrm{t} 3(\mathrm{P}=0.0001)$. B. Percentage of CD34+ cells at $\mathrm{t} 0$ and $\mathrm{t} 3(\mathrm{P}=0.006)$.
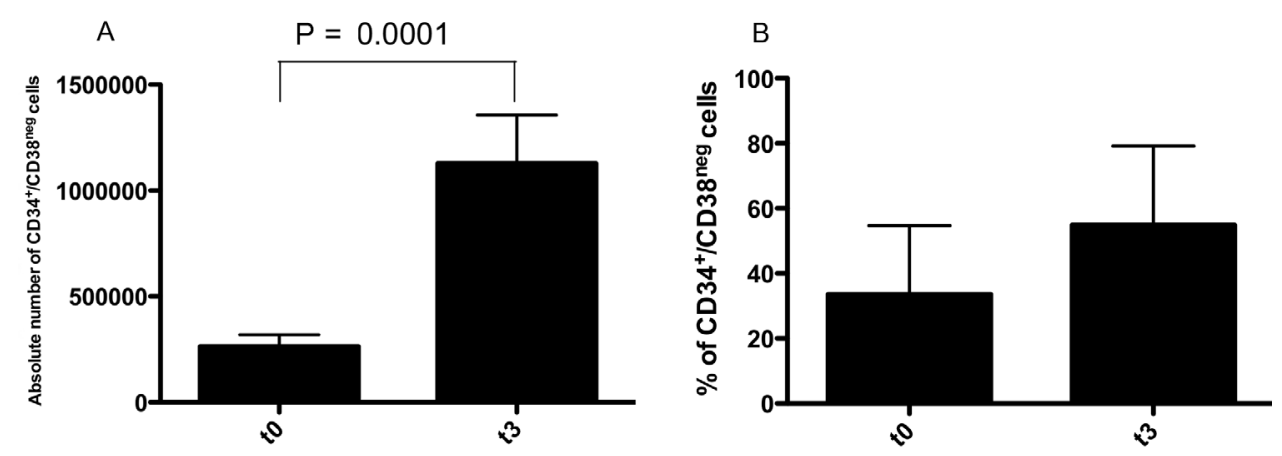

Figure 2. $\mathrm{CD} 4^{+} / \mathrm{CD} 38^{\text {neg }}$ contents in unmanipulated $(\mathrm{t} 0)$ and expanded ( $\left.\mathrm{t} 3\right)$ cells and validation of microarray expression data of t0 and $\mathrm{t} 3$ cells. A. Absolute number of $\mathrm{CD} 34^{+} / \mathrm{CD} 38^{\text {neg }}$ at $\mathrm{t} 0$ and $\mathrm{t} 3(\mathrm{P}=0.0001)$. B. Percentage of $\mathrm{CD} 34^{+} / \mathrm{CD} 38^{-}$cells at t0 and $\mathrm{t} 3(\mathrm{P}=0.012)$.

The top 25 down- and up-modulated genes found in $\mathrm{t} 3$ expanded cells, according to the microarrays, were determined (Tables 1 and 2). Among the top 25 up-regulated genes, there were genes involved in cell division and proliferation, while among down-regulated genes, we 
found modulators of homing, such as chemokine CXCR4 (C-X-C motif receptor 4), HIF1A (hypoxia inducible factor 1 alpha subunit), SERPINA1 (serpin peptidase inhibitor 1), PDE4B (phosphodiesterase 4B, cAMP-specific), and RGS1 (regulator of G-protein signaling 1).

Table 1. Top 25 of negatively regulated genes in expanded cells ( $\mathrm{t} 3$ ) compared to unmanipulated cells ( $\mathrm{t} 0$ ).

\begin{tabular}{llr}
\hline Gene symbol & Description & Fold-change t3/t0 \\
\hline TWF1 & PTK9 protein tyrosine kinase 9 (PTK9) & -502.1 \\
KIFC3 & kinesin family member C3 & -161.2 \\
IFI44L & interferon-induced protein 44-like & -61.9 \\
TPPP3 & tubulin polymerization-promoting, member 3 & -56.7 \\
FBXW7 & F-box and WD-40 domain protein 7 (FBXW7), transcript var. 1 & -51.1 \\
RGS1 & regulator of G-protein signalling 1 & -49.5 \\
CXCL10 & chemokine (C-X-C motif) ligand 10 & -97.9 \\
SIK1 & salt induced kinase 1 & -37.4 \\
SERPINA1 & serine (or cysteine) proteinase inhibitor, member 1 & -24.9 \\
MS4A1 & membrane-spanning 4-domains, subfamily A, member 1 & -19.9 \\
PDE4B & phosphodiesterase E4 dunce homolog, Drosophila & -16.1 \\
ELAVL4 & ELAV (embryonic lethal, abnormal vision, Drosophila)-like 4 & -15.5 \\
VPREB3 & pre-B lymphocyte gene 3 & -15.2 \\
CLEC4E & C-type lectin domain family 4, member E & -13.9 \\
S100A12 & S100 calcium binding protein A12 (calgranulin C) & -13.7 \\
TMSB15A & thymosin beta 15A & -13.6 \\
KLF2 & Kruppel-like factor 2 & -13.3 \\
CD69 & CD69 antigen & -13.3 \\
RAG2 & recombination activating gene 2 & 12.5 \\
HIST2H2BE & histone 2, H2be & -12.5 \\
STK17B & serine/threonine kinase 17b & -12.2 \\
PLBD1 & phospholipase B domain containing 1 \\
XAF1 & XIAP associated factor-, transcript var. 1 & -10.9 \\
DUSP1 & dual specificity phosphatase 1 & -10.8 \\
CXCR4 & chemokine (C-X-C motif) receptor 4 transcript var. 2 & -9.9 \\
& & -9.2 \\
\hline
\end{tabular}

Table 2. Top of 25 positively regulated genes in expanded cells ( $\mathrm{t} 3$ ) compared to unmnaipulated cells ( $\mathrm{t} 0$ ).

\begin{tabular}{llc}
\hline Gene symbol & Description & Fold-change t3/t0 \\
\hline ADAM18 & a disintegrin and metalloproteinase domain 18 & 44.1 \\
PHLDA1 & apoptosis-associated nuclear protein PHLDA1 & 42.1 \\
BBC3 & BCL2 binding component 3 & 33.7 \\
KIF2C & kinesin family member 2C & 24.9 \\
TAS2R1 & taste receptor, type 2, member 1 & 21.5 \\
STAT2 & signal transducer and activator of transcription 2 & 21.1 \\
INMT & indolethylamine N-methyltransferase & 19.7 \\
AFG3L2 & AFG3 ATPase family gene 3-like 2 & 19.6 \\
WNT5B & wingless-type MMTV integration site family, member 5B & 17.7 \\
ITIH1 & inter-alpha (globulin) inhibitor H1 & 17.1 \\
XPO7 & exportin 7 & 14.2 \\
SMAD6 & SMAD, mothers against DPP homolog 6 & 12.1 \\
GAL & galanin & 11.9 \\
PCK2 & phosphoenolpyruvate carboxykinase 2 (mitochondrial) & 11.5 \\
PLK1 & polo-like kinase 1 (Drosophila) & 10.8 \\
PHLDA2 & pleckstrin homology-like domain, family A, member 2 & 10.6 \\
CYLC1 & H. sapiens cylicin mRNA & 10.2 \\
C1orf106 & chromosome 1 open reading frame 106 & 9.3 \\
LAT & linker for activation of T cells & 9.3 \\
MT1F & metallothionein 1F (functional) & 9.1 \\
RBP4 & retinol binding protein 4, plasma & 8.9 \\
AURKB & aurora kinase B & 8.8 \\
IL9R & interleukin 9 receptor (IL9R) & 8.7 \\
HIG2 & hypoxia-inducible protein 2 & 8.5 \\
HBD & hemoglobin, delta & 8.3 \\
\hline
\end{tabular}


Twelve genes were validated by qPCR: five genes of the Wnt pathway: WNT5A, WNT5B, WNT8A (wingless-related MMTV integration site 5A, 5B and 8A), GSK3B (glycogen synthase kinase 3 beta) and DKK1 (dickkopf 1 homolog). Among the down-regulated genes, CXCR4, HOXB4 (homeobox B4) and BMI1 (polycomb ring finger oncogene) were validated. Expression of SMAD6 (SMAD family member 6), a negative regulator of BMP and TGF-beta/activin-signaling that was up-regulated in the expanded cells, was also validated. Finally, cell cycle-related genes, BIRC5 (baculoviral IAP repeat containing 5) and CDC25C (cell division cycle 25 homolog $\mathrm{C}$ ), were also validated and were significantly up-regulated in expanded cells (Figure 3).

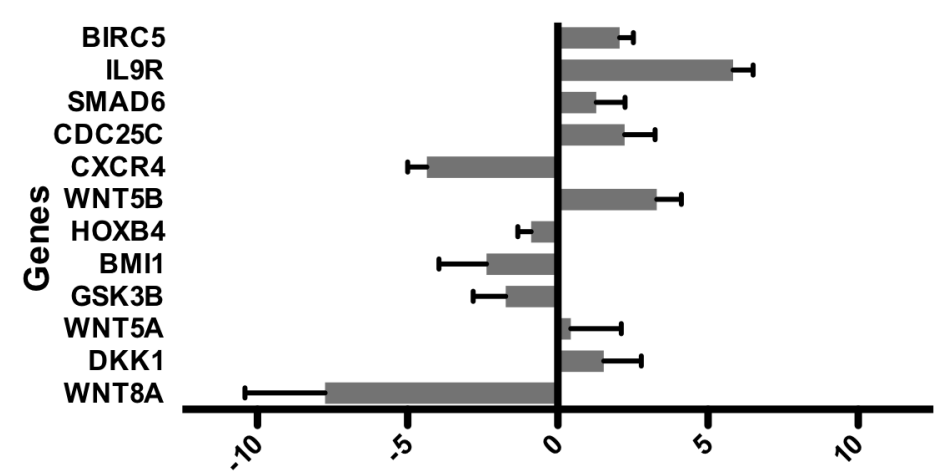

Figure 3. Validation of microarray data by $q P C R$. Y-axis $=$ gene names. $\mathrm{X}$-axis $=$ fold-change values, with t0 as the calibrator samples.

\section{DISCUSSION}

As expected for a short-term expansion culture, the increase in the absolute number of $\mathrm{CD} 34^{+}$cells was modest, albeit significant. Most cells retained expression of the CD34 ${ }^{+}$ marker, and there was a significant increase in the $\mathrm{CD} 34^{+} / \mathrm{CD} 38^{-}$cell population. Despite the known association of the $\mathrm{CD} 34^{+} / \mathrm{CD} 38^{-}$phenotype with a primitive HSC phenotype, it is highly heterogeneous (da Silva et al., 2009).

WNT5B gene expression was increased in expanded HSC, while its paralog, WNT5A, was not significantly altered, suggesting they do not have overlapping functions in this scenario. WNT5B is a noncanonical WNT ligand that inhibits activation of $\beta$-catenin induced by WNT3A (Kanazawa et al., 2005). The only canonical WNT ligand found to be modulated here was WNT8A, which was strongly down-regulated at t3. The down-regulation of canonical ligands and up-regulation of noncanonical ligand WNT5B, WNT receptor FZD9 and canonical inhibitor DKK1, led us to hypothesize that there is a skewing towards noncanonical Wnt signaling during early ex vivo expansion.

The canonical WNT pathway is fundamental for maintenance, expansion and lineage decisions in various tissues, including the hematopoietic system (Jeannet et al., 2008). While canonical WNT pathway is very well defined, regulation of the noncanonical pathway is less clear, with multiple $\beta$-catenin-independent pathways potentially existing side by side (van Amerongen et al., 2008). Regarding the role of canonical WNT pathway in HSC, there are conflicting reports. It has been shown that canonical WNT signaling regulates HSC self-re- 
newal, promoting proliferation and inhibiting differentiation (Kirstetter et al., 2006), but it has also been shown to inhibit self-renewal, depending on the developmental stage (Kokolus and Nemeth, 2010). WNT5A was shown to increase the engraftment of UCB-HSCs by inhibiting WNT3A canonical signals, but it is not known which noncanonical pathways are used by WNT5A (Nemeth et al., 2007). A recent report also showed the importance of noncanonical Wnt for the maintenance of HSC in the niche and in ex vivo cultures in the presence of a supporting osteoprogenitor cell line (Sugimura et al., 2012). These and other conflicting reports reflect different influences and interactions of canonical and noncanonical WNT signals (Fleming et al., 2008), and most importantly, the intrinsic differences between the HSC populations studied and the conditions used in each study. Among these conditions, hypoxia has been recently shown to regulate WNT/ $\beta$-catenin signaling (Kaufman, 2010), and absence of hypoxia during ex vivo expansion could explain the apparent induction of noncanonical WNT signaling. Nevertheless, it is very challenging to interpret WNT signals because they vary considerably depending on the cellular context (van Amerongen and Nusse, 2009).

Taken together, the changes in gene expression during early ex vivo expansion described here suggest that a shift towards noncanonical Wnt signaling is one of the factors involved in the loss of long-term reconstitution by ex vivo expanded UCB-HSC.

\section{ACKNOWLEDGMENTS}

Research supported by the Brazilian agencies FAPESP and CNPq. We thank Maristela Delgado Orellana, Patricia V.B. Palma and Camila Cristina B.O. Menezes for their excellent technical assistance.

\section{REFERENCES}

Astori G, Malangone W, Adami V, Risso A, et al. (2001). A novel protocol that allows short-term stem cell expansion of both committed and pluripotent hematopoietic progenitor cells suitable for clinical use. Blood Cells Mol. Dis. 27: 715-724.

Auvray C, Delahaye A, Pflumio F, Haddad R, et al. (2012). HOXC4 homeoprotein efficiently expands human hematopoietic stem cells and triggers similar molecular alterations as HOXB4. Haematologica 97: 168-178.

Blank U, Karlsson G and Karlsson S (2008). Signaling pathways governing stem-cell fate. Blood 111: 492-503.

da Silva CL, Goncalves R, Porada CD, Ascensao JL, et al. (2009). Differences amid bone marrow and cord blood hematopoietic stem/progenitor cell division kinetics. J. Cell Physiol. 220: 102-111.

Delaney C, Heimfeld S, Brashem-Stein C, Voorhies H, et al. (2010). Notch-mediated expansion of human cord blood progenitor cells capable of rapid myeloid reconstitution. Nat. Med. 16: 232-236.

Fleming HE, Janzen V, Lo CC, Guo J, et al. (2008). Wnt signaling in the niche enforces hematopoietic stem cell quiescence and is necessary to preserve self-renewal in vivo. Cell Stem Cell 2: 274-283.

Gilmore GL, DePasquale DK, Lister J and Shadduck RK (2000). Ex vivo expansion of human umbilical cord blood and peripheral blood CD34+ hematopoietic stem cells. Exp. Hematol. 28: 1297-1305.

Jeannet G, Scheller M, Scarpellino L, Duboux S, et al. (2008). Long-term, multilineage hematopoiesis occurs in the combined absence of beta-catenin and gamma-catenin. Blood 111: 142-149.

Kanazawa A, Tsukada S, Kamiyama M, Yanagimoto T, et al. (2005). Wnt5b partially inhibits canonical Wnt/beta-catenin signaling pathway and promotes adipogenesis in 3T3-L1 preadipocytes. Biochem. Biophys. Res. Commun. 330: 505510 .

Kaufman DS (2010). HIF hits Wnt in the stem cell niche. Nat. Cell Biol. 12: 926-927.

Kelly SS, Sola CB, de Lima M and Shpall E (2009). Ex vivo expansion of cord blood. Bone Marrow Transplant 44: 673-681.

Kirstetter P, Anderson K, Porse BT, Jacobsen SE, et al. (2006). Activation of the canonical Wnt pathway leads to loss of hematopoietic stem cell repopulation and multilineage differentiation block. Nat. Immunol. 7: 1048-1056.

Kokolus K and Nemeth MJ (2010). Non-canonical Wnt signaling pathways in hematopoiesis. Immunol. Res. 46: 155-164.

Genetics and Molecular Research 12 (2): 1691-1697 (2013)

CFUNPEC-RP www.funpecrp.com.br 
McNiece I, Kubegov D, Kerzic P, Shpall EJ, et al. (2000). Increased expansion and differentiation of cord blood products using a two-step expansion culture. Exp. Hematol. 28: 1181-1186.

Nemeth MJ, Topol L, Anderson SM, Yang Y, et al. (2007). Wnt5a inhibits canonical Wnt signaling in hematopoietic stem cells and enhances repopulation. Proc. Natl. Acad. Sci. U. S. A. 104: 15436-15441.

Pfaffl MW (2001). A new mathematical model for relative quantification in real-time RT-PCR. Nucleic Acids Res. 29: e45. Pranke P, Hendrikx J, Debnath G, Alespeiti G, et al. (2005). Immunophenotype of hematopoietic stem cells from placental/ umbilical cord blood after culture. Braz. J. Med. Biol. Res. 38: 1775-1789.

Sugimura R, He XC, Venkatraman A, Arai F, et al. (2012). Noncanonical Wnt signaling maintains hematopoietic stem cells in the niche. Cell 150: 351-365.

van Amerongen R and Nusse R (2009). Towards an integrated view of Wnt signaling in development. Development 136: 3205-3214.

van Amerongen R, Mikels A and Nusse R (2008). Alternative wnt signaling is initiated by distinct receptors. Sci Signal. 1: re9.

Walenda T, Bokermann G, Ventura Ferreira MS, Piroth DM, et al. (2011). Synergistic effects of growth factors and mesenchymal stromal cells for expansion of hematopoietic stem and progenitor cells. Exp. Hematol. 39: 617-628. 